\title{
Estimating Dangerous Areas from Preceding Pedestrian Considering Occlusion Problem
}

\author{
Hiroki Kitamura $^{1}$, Yoshihiro Uemura ${ }^{1}$, Yusuke Kajiwara ${ }^{2}$ and Hiromitsu Shimakawa ${ }^{2}$
}

\begin{abstract}
This paper proposes a method to estimate dangerous areas for visually impaired to walk safely.

In stations and shopping malls, it is difficult to detect dangerous areas because surrounding people shield them, what is called occlusion problem. The method estimates dangerous areas from walking posture of preceding pedestrians. From the result of experiment, the method discriminates between inside and outside dangerous areas from the head and body angles of preceding pedestrians. In addition, it is considered to be possible to distinguish 4 dangerous areas from the change of these angles.
\end{abstract}

Keywords-visually impaired, obstacle, walking support, walking posture, occlusion problem, dangerous areas.

\section{INTRODUCTION}

People get most of their surrounding information through their eyes. Since visually impaired people have no use of vision, they cannot detect obstacles on the street and zones likely to cause fallings. Therefore, they cannot go outside without any walking support. Particularly, they need walking support in stations and shopping malls [1]. As walking support, they use a white cane to detect obstacles and zones likely to cause falls. However, their white cane cannot detect enough obstacles and the zones in stations and shopping malls because surrounding pedestrians in effect shield obstacles and the zones, as it is known as the occlusion problem. Therefore, it is necessary to estimate obstacles and the zones considering the occlusion problem.

The paper estimates positions of obstacles and the zones from walking posture of preceding pedestrians. When preceding pedestrians walk on these zones, they change their walking postures to avoid these zones. For example, when they walk near obstacles on the street, they make a detour to avoid obstacles. When they walk zones likely to cause fallings, they try to take balance, looking the floor. The paper proposes a method to estimate dangerous areas from these walking postures.

\section{RELATED STUDIES}

Many papers have proposed ways to detect obstacles and zones likely to cause falls, such as stairs and differences in level. Many methods use Kinect, super-sonic sensors, and

\footnotetext{
${ }^{1}$ Graduate School of Information Science and Engineering Ritsumeikan University, Shiga, Japan

${ }^{2}$ College of Information Science and Engineering Ritsumeikan University, Shiga, Japan
}

cameras to detect these postures. In the work of Hoang [2], Kinect could detect and warn the person of obstacles to avoid them. In the study of Menikdiwela [3], a white cane equipped with super-sonic sensors could detect obstacles on the street. It helps visually impaired people to avoid obstacles. However, it is difficult to go out with Kinect because Kinect needs high performance calculator and power supply. In addition to that, it is difficult to adapt super-sonic sensors in places with many people, because super-sonic sensors always detect moving obstacles, such as surrounding pedestrians. Therefore, visually impaired cannot walk anywhere. In the study of Tapu [4], a camera in a smartphone could detect and classify obstacles. However, this study did not consider obstacles shielded by the surrounding pedestrians. A method to estimate obstacles and zones shielded by pedestrians is necessary to help visually impaired people to walk outside safely.

\section{ESTIMATION OF DANGEROUS AREAS FROM WALKING BEHAVIOURS}

\section{A. Procedure to Estimate Dangerous Areas}

The paper supposes that visually impaired walk in stations and shopping malls. In these places, dangerous places exist for visually impaired to have risks of collisions or cause fallings. There are some reasons. For example, these places have smooth floors likely to cause falls be slippery when these floors get wet. There are also risks to have collisions with surrounding people, because these places are crowded with many people. Therefore, it is necessary to grasp the positions of obstacles and states on the street. The paper refers to visually impaired as user. The paper defines dangerous areas as obstacles, stairs, and badly placed scaffolding. The paper proposes a method to estimate dangerous areas from the walking posture of preceding pedestrians. Figure 1 shows how to estimate dangerous areas from the walking posture of preceding pedestrians. 


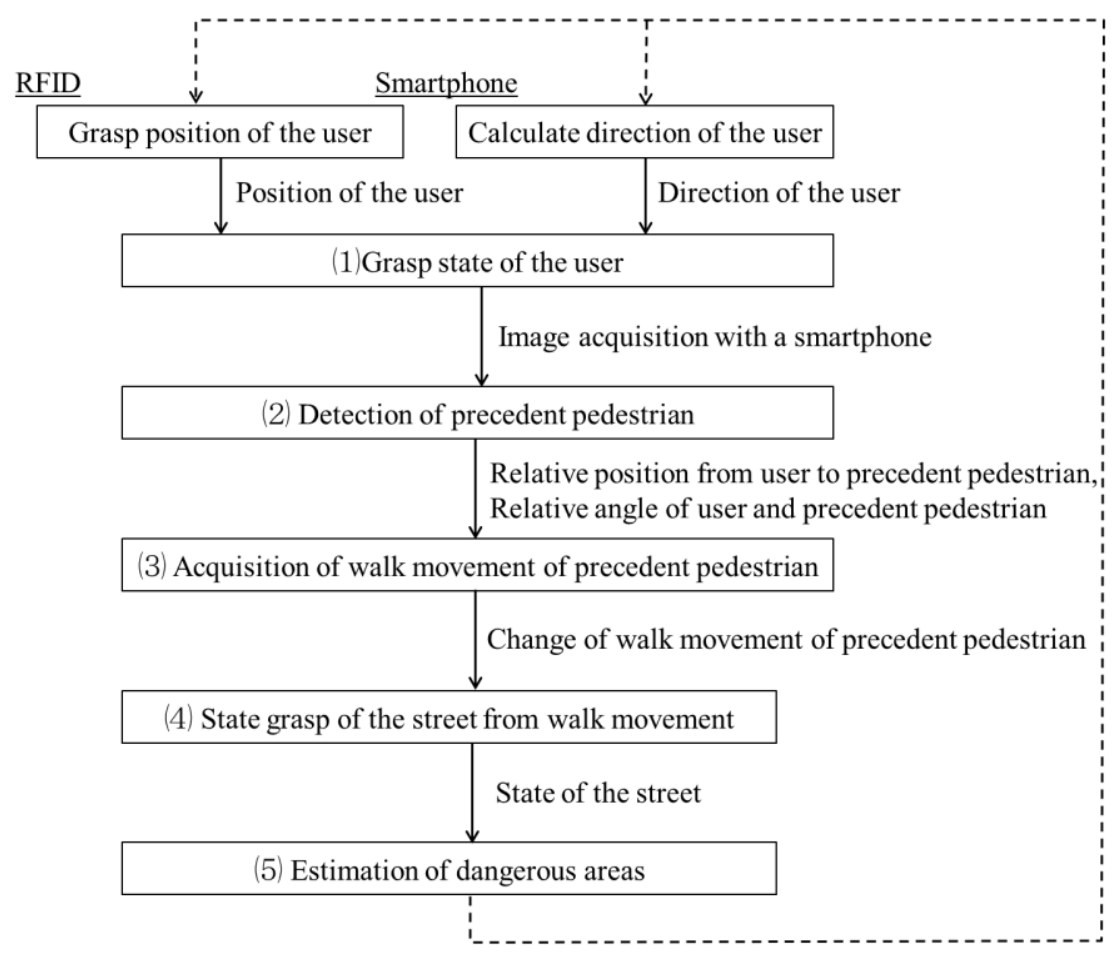

Fig. 1: Estimation of dangerous areas

The method consists of five steps.

1. It grasps the positions of the user who is a visually impaired with RFID technologies. It calculates the directions of the user with a gyro sensor and magnetic field sensor in his smartphone.

2. It detects preceding pedestrians, capturing their images with a smartphone on the user chest.

3. It acquires positions of preceding pedestrians with image processing.

4. It identifies the walking postures of the preceding pedestrians from movements in their head angles and body angles.

5. It estimates dangerous areas from the identified walking posture of the preceding pedestrian.

Through these steps, the method estimates dangerous areas. The visually impaired person can walk safely on the street.

\section{B. States of User and Preceding Pedestrians}

The position and the direction of the user are necessary to estimate dangerous areas. The position and the direction are denoted as $\mathbf{P}_{\mathbf{u t}}$ and $\mathbf{D}_{\mathbf{u t}}$, respectively. $\mathbf{P}_{\mathbf{u t}}$ are acquired using RFID technologies [5]. RFID technologies consist of tags and a reader, where the RFID tags are placed on the floor and the RFID reader is attached to the user's foot (Figure 2).

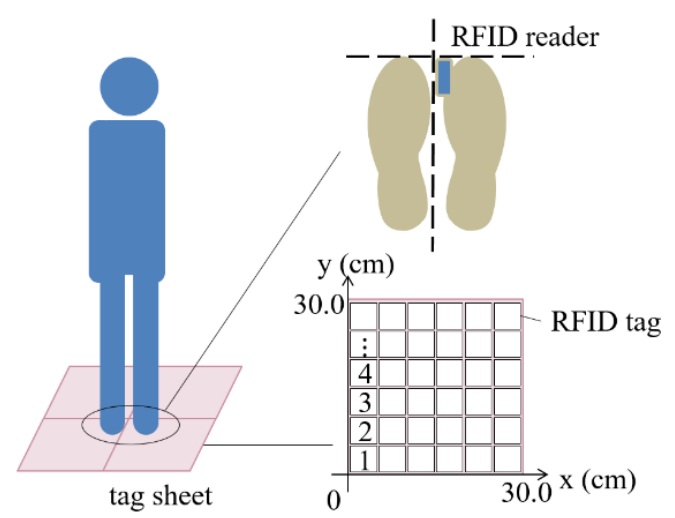

RFID technologies can acquire $\mathbf{P}_{\mathbf{u t}}$ accurately, because the surrounding obstacles do not block radio waves [6]. $\mathbf{D}_{\mathbf{u t}}$ is calculated with a gyro sensor and a magnetic field sensor in user's smartphone attached at the user's chest. The negative direction of the $\mathrm{z}$-axis is defined as walking direction of the user. The positive direction of the $\mathrm{x}$-axis is defined as the rightward of the user. Positive direction of the y-axis is defined as the direction from the floor to the ceiling. The origin

$\mathbf{0}(\mathbf{0}, \mathbf{0}, \mathbf{0})$ is regarded as the position of the camera in his smartphone.

Next, the method detects preceding pedestrians in images in front of the user. After detecting preceding pedestrians, the 
method acquires their walking posture. In the method, their walking posture is represented with the angles of the head and body of a preceding pedestrian. It is possible to estimate dangerous areas from changes in the angles recorded when preceding pedestrians avoid the dangerous areas. For example, when they avoid an obstacle on the street, their y-axis angles of the body change because of the avoidance. The method estimates dangerous areas from these changes in the angles.

\section{EXPERIMENT}

\section{A. Environment for experiment}

Changes in angles of the head and the body of preceding pedestrians are examined in four kinds of dangerous areas. Figure 3 shows the experiment environment. The four dangerous areas are obstacles on the street (dangerous area V), obstacles at high position (dangerous area $\mathrm{X}$ ), up and down stairs (dangerous area Y), and badly placed scaffolding (dangerous area $\mathrm{Z}$ ). In this experiment, five subjects walk six times of each route in Figure.3. They attach RFID reader at their right tow to acquire their positions showed to Figure 2.

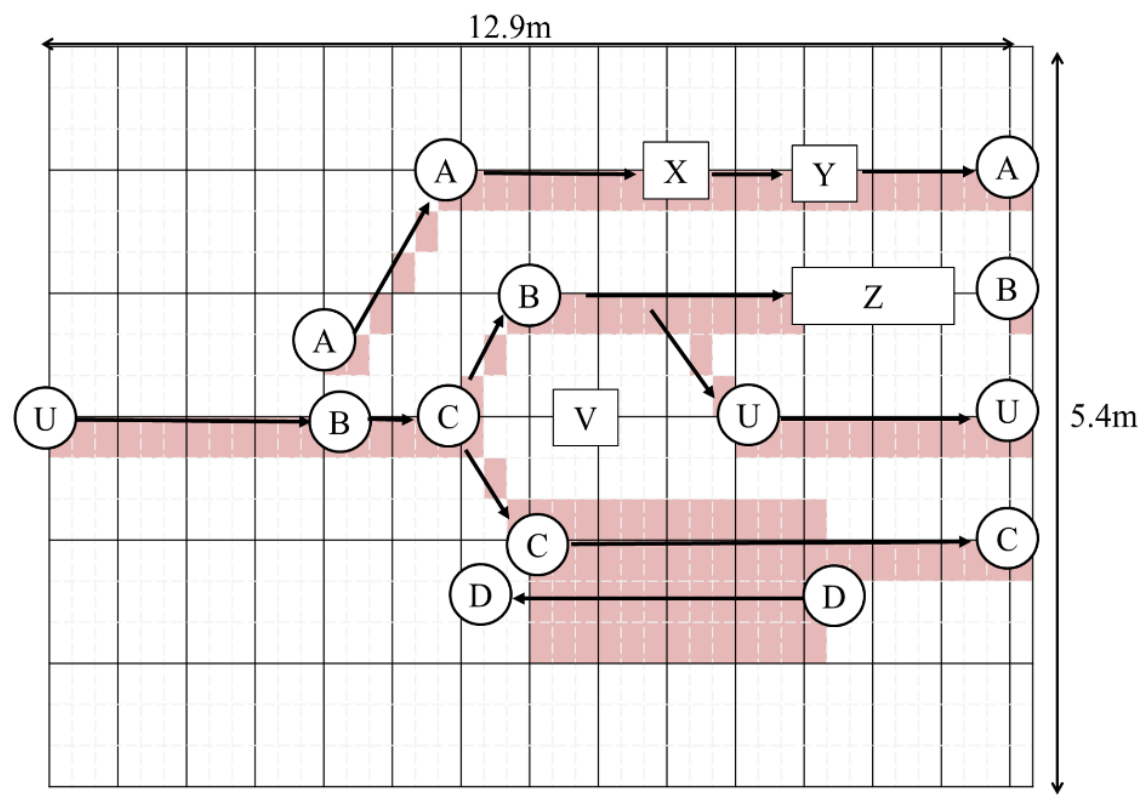

$\rightarrow$ :Route U:Visually impaired people A D:Healthy pedestrians $: \underline{\text { RFID tag sheet }}$

$\mathrm{V}$ : Obstacle on the street. $(104 \mathrm{~cm} \times 59 \mathrm{~cm} \times 100 \mathrm{~cm}) X$ : Mind your head. $(22 \mathrm{~cm} \times 21 \mathrm{~cm} \times 22 \mathrm{~cm})$

$\mathrm{Y}$ : Walking up and down staircases. $(60 \mathrm{~cm} \times 40 \mathrm{~cm} \times 60 \mathrm{~cm}) \mathrm{Z}$ : Mind your step. $(60 \mathrm{~cm} \times 270 \mathrm{~cm})$

Fig. 2: Experiment Environments

\section{B. Calculation}

To estimate dangerous areas, six kinds of data are acquired:

- $P_{u t}\left(x_{u t}, y_{u t}, z_{u t}\right):$ Absolute position of the user.

- $P_{i t}\left(x_{i t}, y_{i t}, z_{i t}\right)$ : Relative position from the user to preceding pedestrians.

- $P_{i t}^{y}\left(x_{i t}, y_{i t}, z_{i t}\right)$ : Absolute position of preceding pedestrians.

- $D_{u t}\left(\alpha_{u t}, \beta_{u t}, \gamma_{u t}\right)$ : Absolute directions of the user.

- $D_{i t}\left(\alpha_{i t}, \beta_{i t}, \gamma_{i t}\right)$ : Relative directions from the user to preceding pedestrians.

- $D_{i t}^{t}\left(\alpha_{i t}, \beta_{i t}, \gamma_{i t}\right)$ : Absolute directions of preceding pedestrians, where $x_{n}, y_{n}$, and $z_{n}$ mean $x, y$, and $z$ coordinate in $P_{n}$, respectively, while $\alpha_{n}, \beta_{n}$, and $\gamma_{n}$ correspond to the angle around $\mathrm{x}^{-}, \mathrm{y}^{-}$, and $\mathrm{z}$-axis in $D_{n}$, respectively.
$P_{w t}$ is calculated with RFID. $P_{i t}$ and $D_{i t}$ are calculated with ARtoolkit [7]. ARtoolkit can acquire the model view matrix ( $M_{i t}$ ) of each marker. $M_{i t}$ is described below. For simplification, $\sin \theta, \cos \theta$, and $\tan \theta$ are represented with $s \theta, c \theta$, and $t \theta$, respectively.

$M_{i t}=\left(\begin{array}{cccc}c \beta_{i t} c \gamma_{i t} & s \alpha_{i t} s \beta_{i t} c \gamma_{i t}+c \alpha_{i t} c \gamma_{i t} & -c \alpha_{i t} s \beta_{i t} c \gamma_{i t}+s \alpha_{i t} s \gamma_{i t} & x_{i t} \\ -c \beta_{i t} s \gamma_{i t} & -s \alpha_{i t} s \beta_{i t} s \gamma_{i t}+c \alpha_{i t} c \gamma_{i t} & c \alpha_{i t} s \beta_{i t} s \gamma_{i t}+s \alpha_{i t} c \gamma_{i t} & y_{i t} \\ s \beta_{i t} & -s \alpha_{i t} c \beta_{i t} & c \alpha_{i t} c \beta_{i t} & z_{i t} \\ 0 & 0 & 0 & 1\end{array}\right)$

AR marker attached to the head and the body of each preceding pedestrian enables us to acquire angles in the head and the body of preceding pedestrians as $D_{i t \text {. }} P_{i t}$, and $D_{i t}$, are calculated from $M_{i t}$, component as follows:

- $P_{\text {it }}:$ in the 4 th column.

- $\alpha_{i t}$ : in the 3rd row and the 3rd column, and in the 3rd row and the 2 nd.

- $\beta_{i t}$ : in the 3rd row and the 1 st column. 
- $\gamma_{i t}$ : in the 2 nd row and the 1 st column, and in the 1 st row and the 1 st.

- $P_{i t}^{\prime}$ is calculated with following formula $P_{i t}^{\prime}=P_{u t}+\left(\begin{array}{ccc}1 & 0 & 0 \\ 0 & c \alpha_{u t} & -s \alpha_{u t} \\ 0 & s \alpha_{u t} & c \alpha_{u t}\end{array}\right)\left(\begin{array}{ccc}c \beta_{u t} & 0 & -s \beta_{u t} \\ 0 & 1 & 0 \\ s \beta_{u t} & 0 & c \beta_{u t}\end{array}\right)\left(\begin{array}{ccc}c \gamma_{u t} & -s \gamma_{u t} & 0 \\ s \gamma_{u t} & c \gamma_{u t} & 0 \\ 0 & 0 & 1\end{array}\right) P_{i t}$ where $D_{i t}^{s}$ is calculated from difference of $D_{i t}$ and $D_{u t}$

\section{Result of Mann Whitney U test}

We examine various angles by the Mann Whitney U test. As for the null hypothesis, we adopted these average angles inside and outside dangerous areas do not show differences. Significance level is defined as 5\%. Table 1 indicates the results of the Mann Whitney $U$ test. Table 1 shows that significant differences in angles of the head and body appear at every dangerous area. The result reveals that some angles acquired in dangerous areas are not identical to some angles acquired in other areas. Therefore, it is possible to distinguish whether a place is dangerous areas or not, from some angles appeared in significant differences.

TABLE I. Result OF The MANN Whitney U Test

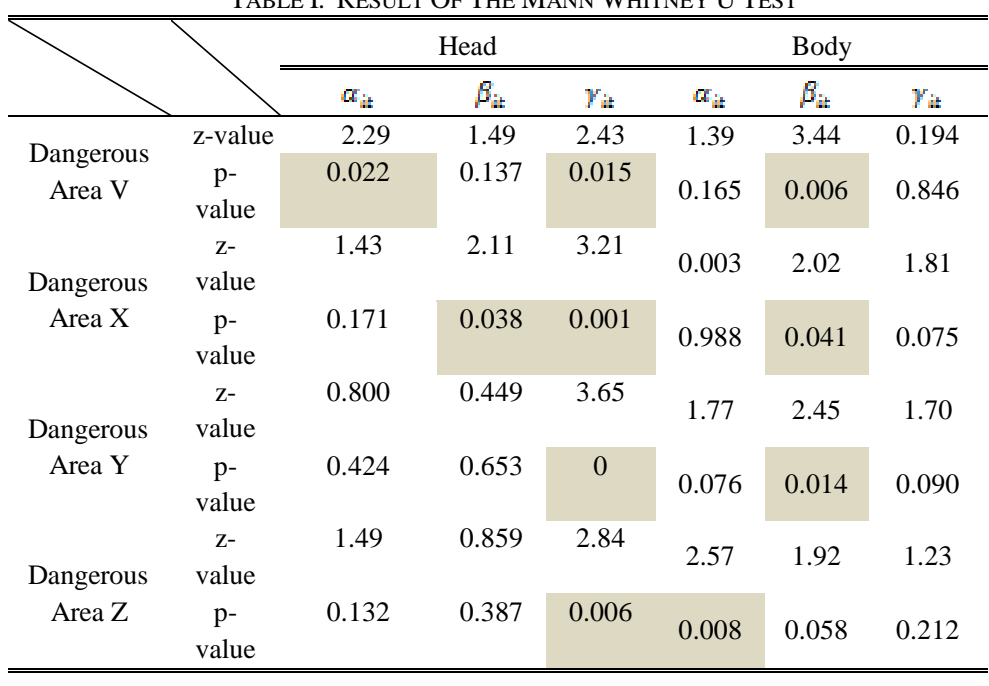

\section{DISCUSSION}

The results of the Mann Whitney $U$ test reveal that significant differences appear in several angles during movement of the head and body in dangerous areas. In addition, the differences vary with each kind of dangerous areas. Therefore, we consider features of walking posture when preceding pedestrians pass or avoid dangerous areas. The features confirm from changes of angles of the head and body $\left(D_{i t}\right)$ appeared significant differences. Furthermore, we consider whether dangerous area $\mathrm{V}$ and dangerous area $\mathrm{X}$ can be distinguished from the features. Each dangerous area is divided to 10 points to confirm changes of angles. The each point is defined as $n$. We consider the feature from difference from $D_{i t}$ in dangerous areas to $D_{i t}$ in the other areas.

$D_{i t}$ of the head is defined as $D_{i t}\left(\alpha_{\text {hit }}, \beta_{\text {hit }}, \gamma_{\text {hit }}\right) . D_{i t}$ of the body is defined as $D_{i t}\left(\alpha_{\text {bit }}, \beta_{\text {bit }}, \gamma_{\text {bit }}\right)$. Table 1 shows that $\alpha_{\text {hit }}, \gamma_{\text {hit }}$, and $\beta_{\text {bit }}$ appear significant differences in dangerous area $\mathrm{V}$. We consider feature of walking posture from difference between $\beta_{\text {bit }}$ in dangerous area $\mathrm{V}$ and $\beta_{\text {bit }}$ in the other areas.

Figure 4 shows difference and variance of $\beta_{\text {bit }}$ in dangerous areas V. $\beta_{\text {bit }}$ take about 20 degrees from $n=0$ to $n=5$ and 5 degrees from $n=6$ to $n=8$. This changes indicate that preceding pedestrian changes his body direction to left from $n=0$ to $n=5$. He also changes his body to the front from $n=6$ to $n=8$. This change represents that preceding pedestrian makes a detour when he avoids obstacles on the street. Therefore, we can estimate dangerous area $\mathrm{V}$ from preceding pedestrians of making a detour. The changes of variance from $n=1$ to $n=2$ and from $n=4$ to $n=5$ are larger than other changes. In these points, preceding pedestrians start or finish avoiding obstacles on the street. Therefore, the point which preceding pedestrians starts or finishes avoiding is different.
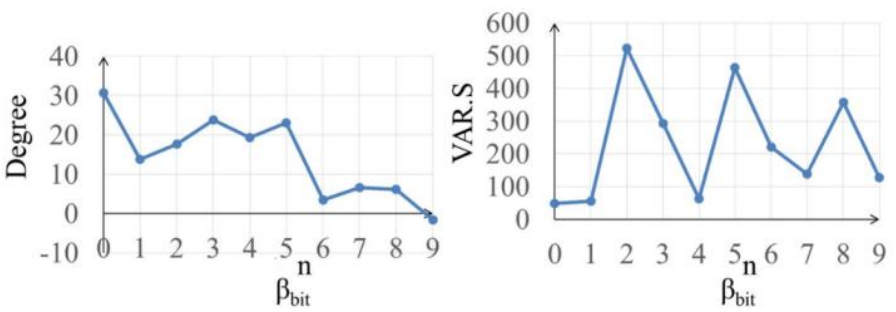

Fig. 3: Difference and variance of $\beta_{\text {bit }}$ in dangerous area $\mathrm{V}$ and $\beta_{\text {bit }}$ in the other areas
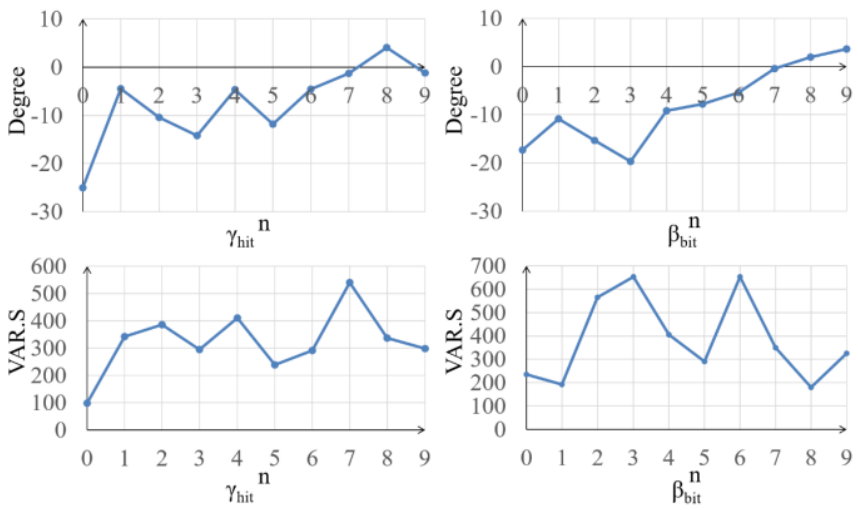

Fig. 4: Difference and variance of $\gamma_{\text {hit }}$ and $\beta_{\text {bit }}$ in dangerous area $X$

Table 1 shows that $\beta_{\text {hit }}, \gamma_{\text {hit }}$, and $\beta_{\text {bit }}$ appear significant differences in dangerous area $\mathrm{X}$. We consider feature of walking posture from difference between $\gamma_{\text {hit }}$ and $\beta_{\text {bit }}$ in dangerous area $\mathrm{X}$ and $\gamma_{\text {hit }}$ and $\beta_{\text {bit }}$ in the other areas, respectively.

Figure 5 shows difference and variance of $\gamma_{\text {hit }}$ and $\beta_{\text {bit }}$ in dangerous areas V. $\gamma_{\text {hit }}$ takes negative degrees from $\mathrm{n}=0$ to $\mathrm{n}=7$, and $\mathrm{n}=9$. $\gamma_{\text {hit }}$ takes positive degrees at $\mathrm{n}=8$. This represents that preceding pedestrians put his head on the right side in the most range. $\beta_{\text {bit }}$ takes negative degrees from $n=0$ to $n=6$ and positive degrees from $\mathrm{n}=7$ to $\mathrm{n}=9$. These changes indicate that preceding pedestrian changes his body direction to right from $n=0$ to $n=6$. After that, he changes his body 
direction to the left a little. The obstacle at high position is set near the point of $n=6$. Therefore, the preceding pedestrian makes a detour to avoid obstacles at as high position as on the street. Furthermore, he puts his head on the right side to avoid obstacles, because the obstacles exist at high position. Variance of $\gamma_{\text {hit }}$ represents the maximum value at $\mathrm{n}=7$. Variance of $\beta_{\text {bit }}$ represents the maximum value at $n=3$ and $\mathrm{n}=7$. Therefore, the positons that preceding pedestrians start or finish avoiding obstacles at high position vary with each preceding pedestrians.

Figure 4 and figure 5 indicate that preceding pedestrians change their body direction to make a detour in order to avoid obstacles in dangerous area $\mathrm{X}$ and dangerous area V. However, walking posture that preceding pedestrians put their head on the right side appears only in dangerous area V. Therefore, these dangerous areas are distinguished from walking posture of $\gamma_{\text {hit }}$.

\section{CONCLUSION}

This paper proposes a method to estimate dangerous areas for visually impaired to walk safely in places where many people go across. We examine whether it is possible to distinguish dangerous areas from non-dangerous ones with changes of the angle of the head and the body. As a result of the Mann Whitney U test, significant differences in parts of these angles appear in every dangerous area. Therefore, the method estimates dangerous areas from these angles.

As future works, we will examine whether significant differences in these angles appear at other kinds of dangerous areas. Using the proposed method, visually impaired people can walk alone even if they are not accompanied by guiding people.

\section{REFERENCES}

[1] http://www.mhlw.go.jp/bunya/shougaihoken/cyousajigyou/jiritsushien\_ project/seillka/research \_09/d1/result/08-09a.pdf

[2] Hoang, Van-Nam, et al. "Obstacle detection and warning for visually impaired people based on electrode matrix and mobile Kinect." Information and Computer Science (NICS), 2015 2nd National Foundation for Science and Technology Development Conference on. IEEE, 2015. 4)

http://dx.doi.org/10.1109/nics.2015.7302222

[3] Menikdiwela, M. P., and K. M. I. S. Dharmasena. "Haptic based walking stick for visually impaired people." Circuits, Controls and Communications (CCUBE), 2013 International conference on. IEEE, 2013.5)

http://dx.doi.org/10.1109/ccube.2013.6718549

[4] Tapu, Ruxandra, et al. "A smartphone-based obstacle detection and classification system for assisting visually impaired people." Proceedings of the IEEE International Conference on Computer Vision Workshops. 2013.

http://dx.doi.org/10.1109/iccvw.2013.65

[5] Uemura, Yoshihiro, et al. "Estimating Human Physical States from Chronological Gait Features Acquired with RFID Technology." Sensors \& Transducers 194.11 (2015): 76
[6] Wong, Wallace, et al. "Accurate indoor positioning technique using RSSI assisted inertial measurement." Future Information Communication Technology and Applications. Springer Netherlands, 2013. 121-129.

http://dx.doi.org/10.1007/978-94-007-6516-0_14

[7] Kato, Hirokazu, et al. "A registration method based on texture tracking using artoolkit." Augmented Reality Toolkit Workshop, 2003. IEEE International. IEEE, 2003 http://dx.doi.org/10.1109/art.2003.1320435 